\title{
Study of the Quantum Confinement Effects and Stability Properties of Small Nanoclusters of Bare and Hydrogenated Diamond
}

\author{
M. Yeganeh ${ }^{a, *}$, F. Badieian Baghsiyahi ${ }^{a}$ And R. Pilevar Shahri ${ }^{b}$ \\ ${ }^{a}$ School of Natural Science, Kosar University of Bojnord, P.O. Box 94104455, Bojnord, Iran \\ ${ }^{b}$ Department of Physics, Payame Noor University (PNU), P.O. Box 19395-3697, Tehran, Iran \\ (Received December 3, 2018; revised version April 10, 2019; in final form May 2, 2019)
}

\begin{abstract}
Density functional theory, as implemented in SIESTA code, was utilized in this study to investigate the structural, electronic and stability properties of bare and hydrogenated small nanoclusters of diamond. The results obtained by studying different nanoparticles of diamond composed of 19, 50, and 104 carbon atoms, revealed that while the gap energy of hydrogenated nanodiamonds reduced from 8.2 to $6.5 \mathrm{eV}$ by increasing the size of nanoparticles (number of carbon atoms), the bare nanodiamonds showed almost no gap energy except $\mathrm{C}_{19}$ sample which has the highest occupied molecular orbital-lowest unoccupied molecular orbital of about 0.33 eV. Electron affinity of hydrogenated samples was calculated and it was found that hydrogenated nanodiamond exhibits negative electron affinity. The quantum confinement effect found to be still significant for the sample larger than $1 \mathrm{~nm}$ i.e. the largest hydrogenated sample of $\mathrm{C}_{104} \mathrm{H}_{90}$ with a diameter of about $1.2 \mathrm{~nm}$ which showed greater gap energy in comparison to the bulk diamond. This achievement was explained considering electron affinity and partial density of states analysis. The calculated formation energy of the nanoparticles confirmed that the small hydrogenated nanoclusters of diamond have more stability compared to the bare nanodiamonds.
\end{abstract}

DOI: 10.12693/APhysPolA.136.151

PACS/topics: nanodiamond, structural and electronic properties, electron affinity, quantum confinement effects, structural stability

\section{Introduction}

Diamond based nanocrystalline materials synthesized by different methods such as chemical vapor deposition (CVD) and detonation techniques having particles with diameter in the range of $1-100 \mathrm{~nm}$ are called nanodiamond (ND) [1, 2]. Nanodiamond in its pure (bare) form exhibits core-shell bucky diamond structure with the diamond-like core and fullerenic outer shell. In this case the band gap of diamond is filled by the states from the graphitic components [1]. Addition of chemical groups to the surface of nanoparticles known as functionalization, is interesting for the experimental and theoretical researches. Functionalization of ND is not only important regarding the stability of nanodiamond against graphitization but also can control other properties of nanodiamond [3]. By considering the large surface to the volume ratio of nanoparticles, modification and functionalization of nanodiamond surface can strongly influence its bulk properties.

Functionalization of nanodiamond by hydrogen or "hydrogenation" of the surface can stabilize the surface of nanodiamond $[1,4]$. As a consequence of surface hydrogenation of nanodiamond, surface structure becomes similar to the bulk diamond $[5,6]$. Indeed, saturation of the surface by hydrogen prevents the nanodiamond to

\footnotetext{
* corresponding author; e-mail: mahboubeh. yeganeh@yahoo.co.uk
}

form onion-like carbon or bucky diamond. The structure of bare nanodiamond consists of deformed $\sigma$ and $\pi$ bonding, but the hydrogenated particles show only the $\sigma$ bonds [5].

Small nanoclusters having diameter below $1 \mathrm{~nm}$, are of great interest for applications of catalysts, molecular machine component, nanoelectronic devices, and biosensors [7]. They exhibit different properties from the bulk as well as the larger nanoparticles [7]. Considering the importance of this category of nanoclusters, the synthesis of small nanoclusters is the first important issue. In this regard nanoclusters of diamond as small as $1.1 \mathrm{~nm}$ were synthesized by Stehlik et al. [8]. Further more, they provided experimental evidence for stability of diamond smaller than $1 \mathrm{~nm}$. Upon decreasing nanoparticles size to a critical value, their structural stability and physical properties are influenced by quantum confinement effects [9]. Although many experimental and theoretical studies were performed to investigate the quantum confinement effects on electronic structure and stability of nanodiamond in one side and to predict the critical size in which the quantum confinement effects starts to develop on the other side, but controversial results were reported in literature. Chang et al. [9] studied the quantum confinement effects on a series of nanodiamond thin films with particle diameters ranging from $3.5 \mathrm{~nm}$ to $5 \mu \mathrm{m}$ by X-ray absorption spectroscopy. They observed that the conduction band shifted toward higher energy when the particle diameter was decreased. The gap energy widening was especially detected when the crystallite radius was smaller than $18 \mathrm{~nm}$. 
Raty et al. [10] theoretically investigated the stability of bare and hydrogenated diamond with generalize gradient approximation (GGA) and they found that for particles between 2 and $3 \mathrm{~nm}$, the hydrogenated diamond are less favored energetically in comparison to the bare diamond. Saani et al. [11] used B3LYP exchange-correlation functional to investigate the stability and electronic properties of spherical and cage-like structure of hydrogenated nanodiamond. They showed that the spherical shapes of nanodiamond are more stable than the cage-like structures. In spherical structures, the gap energy increased from 7.9 to $10.6 \mathrm{eV}$ and the formation energy decreased from -9.24 to $-9.81 \mathrm{eV}$ when the number of carbon atoms that make up the nanoparticles, decrease from 47 to 5 atoms.

Wang et al. [3] studied the bare nanodiamond consisting of $66,147,275$, and 476 atoms by GGA. They observed no visible gap energy while the cohesive energy changed from -8.18 to $-8.83 \mathrm{eV}$ for $\mathrm{C}_{66}$ to $\mathrm{C}_{275}$. In another attempt, Wang et al. [12] investigated a wide variety of hydrogenated diamond containing 10 up to 147 carbon atoms by the Perdew-Burke-Ernzerhof (PBE) functional. The variations of HOMO, LUMO, and gap energies were investigated in their work. The gap energy was altered from about $5 \mathrm{eV}$ for $\mathrm{C}_{147} \mathrm{H}_{100}$ to $7.5 \mathrm{eV}$ in case of $\mathrm{C}_{10} \mathrm{H}_{16}$. Jiang et al. [13] reported the gap energy variation from about 5 to $7 \mathrm{eV}$ when the size of hydrogenated diamond changed from 1 to about $0.5 \mathrm{~nm}$ by using GGA approximation whereas by applying local density approximation (LDA), the gap energy changed from about 5.8 to $8.2 \mathrm{eV}$ for the same particles size. The optical properties of hydrogen terminated diamond containing 10 to 87 carbon atoms were investigated theoretically employing PBE functional by Drummond et al. [14] in which the quantum confinement effects were vanished in particles larger than $1 \mathrm{~nm}$.

It can be noticed that controversial results were presented in the literature while in some studies the widening of the gap energy in comparison to the gap energy of bulk diamond $(\approx 5 \mathrm{eV})$ could not be observed for particles larger than $1 \mathrm{~nm}[10,12]$. In some other studies, the widening of gap energy was still significant for particles with radius larger than $18 \mathrm{~nm}$ [9]. Due to the importance of small nanoclusters of ND, as the wide band gap materials, in new developed materials and applications such as field emitters [12], optoelectronic devices [15, 16], and biosensors [17], it seems to be critical to investigate the stability and quantum confinement effects of the particles smaller than or in the range of $1 \mathrm{~nm}$. As the bare nanodiamonds have been less investigated, we have constructed three configurations of bare nanodiamond smaller than $1 \mathrm{~nm}$ by 19,50 , and 104 carbon atoms (called by $\mathrm{C}_{19}$, $\mathrm{C}_{50}$, and $\mathrm{C}_{104}$ ) and then their stability and quantum confinement effects were investigated by DFT. As it has been revealed by the experimental reports, the nanodiamonds have almost spherical shape [18-20]. Therefore, it is desired to construct spherical shape of nanodiamond for theoretical calculations. In this regard, the minimum energy or the Wulff shapes of nanodiamond by Wulff construcion method [21] through the public domain software of Wulffman [22] were investigated by DFT. The rough approximation to the spherical ND shape was achieved considering equal surface energy of $1 \mathrm{eV}$ for [100], [110], and [111] facets by Wulff construction method at various radius of 3,4 , and $5 \AA$.

DFT calculations were performed on the samples having the same number of carbon, but fully hydrogenated $\left(\mathrm{C}_{19} \mathrm{H}_{30}, \mathrm{C}_{50} \mathrm{H}_{66}\right.$, and $\left.\mathrm{C}_{104} \mathrm{H}_{90}\right)$, in order to be able to compare the electronic and stability properties of these two kinds of small nanoclusters of diamond. Besides considering the minimum energy shapes of ND in this work, in order to have better understanding of the electronic properties, the density of states (DOS) and partial density of states (PDOS) analysis of the samples, which have been less noticed in previous works, were discussed. Taking the achieved results in literature into consideration and using the spherical shape of ND which has been known stable in experimental and theoretical results [1], the electronic and stability properties of both hydrogenated and bare nanodiamonds were investigated and the presence of the quantum confinements in our studies were explained. The carbon-carbon bond length variations as a function of distance from the center of nanodiamond were also studied.

\section{Computational method}

Structural and electronic properties of bare and hydrogenated nanodiamond were investigated by density functional theory (DFT) implemented in SIESTA code which is categorized in the atom-centered basis sets methods and is suitable for study the small nanoparticles and molecules [23]. Exchange correlation was defined by GGA approximation via functional theory proposed by Perdew, Burke and Ernzerhof [24]. The valence electrons interactions to the ionic core were approximated by norm-conserving pseudopotentials through the Troullier and Martins method [25, 26].

The valence configurations were constructed by double- $\zeta$ numerical atomic orbitals basis sets. The Brillouin zone was adjusted by $10 \times 10 \times 10 k$-points in the Monkhorst-pack scheme [27]. In order to avoid possible interactions between two adjacent particles, the vacuum distance larger than $10 \AA$ was applied in all 3 directions $(x, y$, and $z)$. The atomic structures were fully optimized using the conjugate gradient (CG) method until the force on each ion is reduced to below $0.01 \mathrm{eV} / \AA$.

\section{Results and discussion}

Figure 1 illustrates the structure of bare and hydrogenated nanodiamond after geometrical optimization. The bond lengths of carbon-carbon $(\mathrm{C}-\mathrm{C})$ of different bare nanodiamond samples were illustrated as a function of distance from the cluster center in Fig. 2. The number of closest carbonic neighbors of carbon atoms was also 

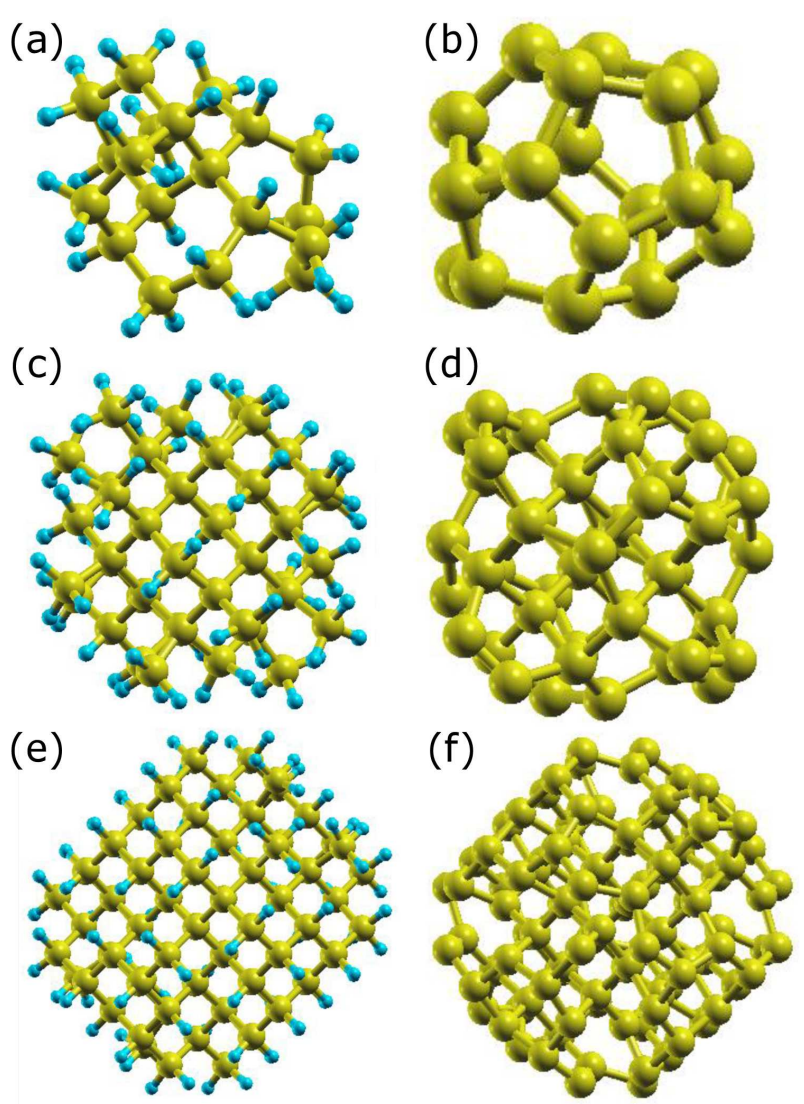

Fig. 1. The geometrical optimized structure of (a) $\mathrm{C}_{19} \mathrm{H}_{30}$, (b) $\mathrm{C}_{19}$, (c) $\mathrm{C}_{50} \mathrm{H}_{66}$, (d) $\mathrm{C}_{50}$, (e) $\mathrm{C}_{104} \mathrm{H}_{90}$ and (f) $\mathrm{C}_{104}$ (carbon and hydrogen are presented in yellow and cyan, respectively).

depicted in Fig. 3. As it has been described in literature $[12,28]$, the $\mathrm{C}-\mathrm{C}$ bond length of about $1.4 \AA$ and $1.54 \AA$ are close to the values of $s p^{2}$ and $s p^{3}$ bonds, respectively. The mean value for the $\mathrm{C}-\mathrm{C}$ bond length of the carbon atoms located at the interface of the core and surface of the nanoparticles was estimated to be about $1.6 \AA[12]$. This kind of bond is longer than $\mathrm{C}-\mathrm{C}$ bond length of $s p^{3}$ in bulk diamond and is highly likely to be created as a consequence of the effective tensile stress between reconstructed diamond surface and core [29]. The $\mathrm{C}-\mathrm{C}$ bond length variations as a function of distance from the center of nanodiamond in this work, depicted in Fig. 2, show that the overall patterns of the bond lengths are almost consistent with the literature. However, the $\mathrm{C}-\mathrm{C}$ bond length at the interface of the core and the surface of the nanocluster of $\mathrm{C}_{104}$ were estimated between 1.7 and $1.8 \AA$.

All $\mathrm{C}-\mathrm{C}$ bonds existed in hydrogenated nanodiamond samples consist of $s p^{3}$ bonds, while the $s p^{2}$ bonds can also be observed in optimized bare nanodiamond. In agreement to the literature $[1,3]$, the core of the bare ND particles often kept their diamond-like structures, while the atoms located at or close to the surface were distributed much disorderly into fullerene-like structures.

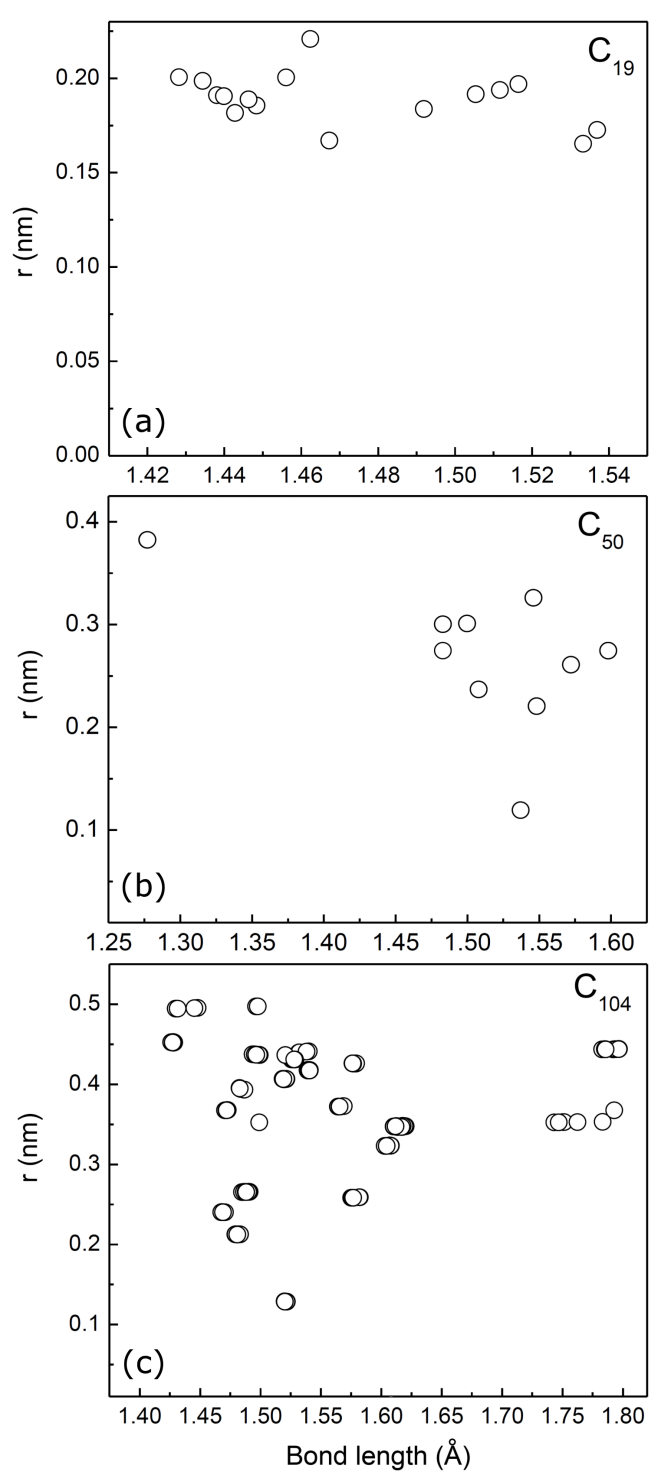

Fig. 2. The bonds length of $\mathrm{C}-\mathrm{C}$ in bare nanodiamonds as a function of distance to the cluster center.

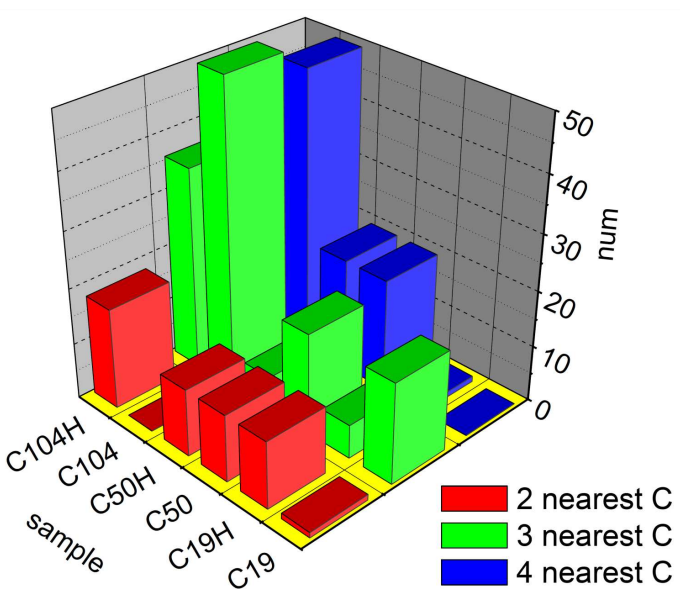

Fig. 3. The number of nearest carbonic neighbors in bare and hydrogenated samples. 

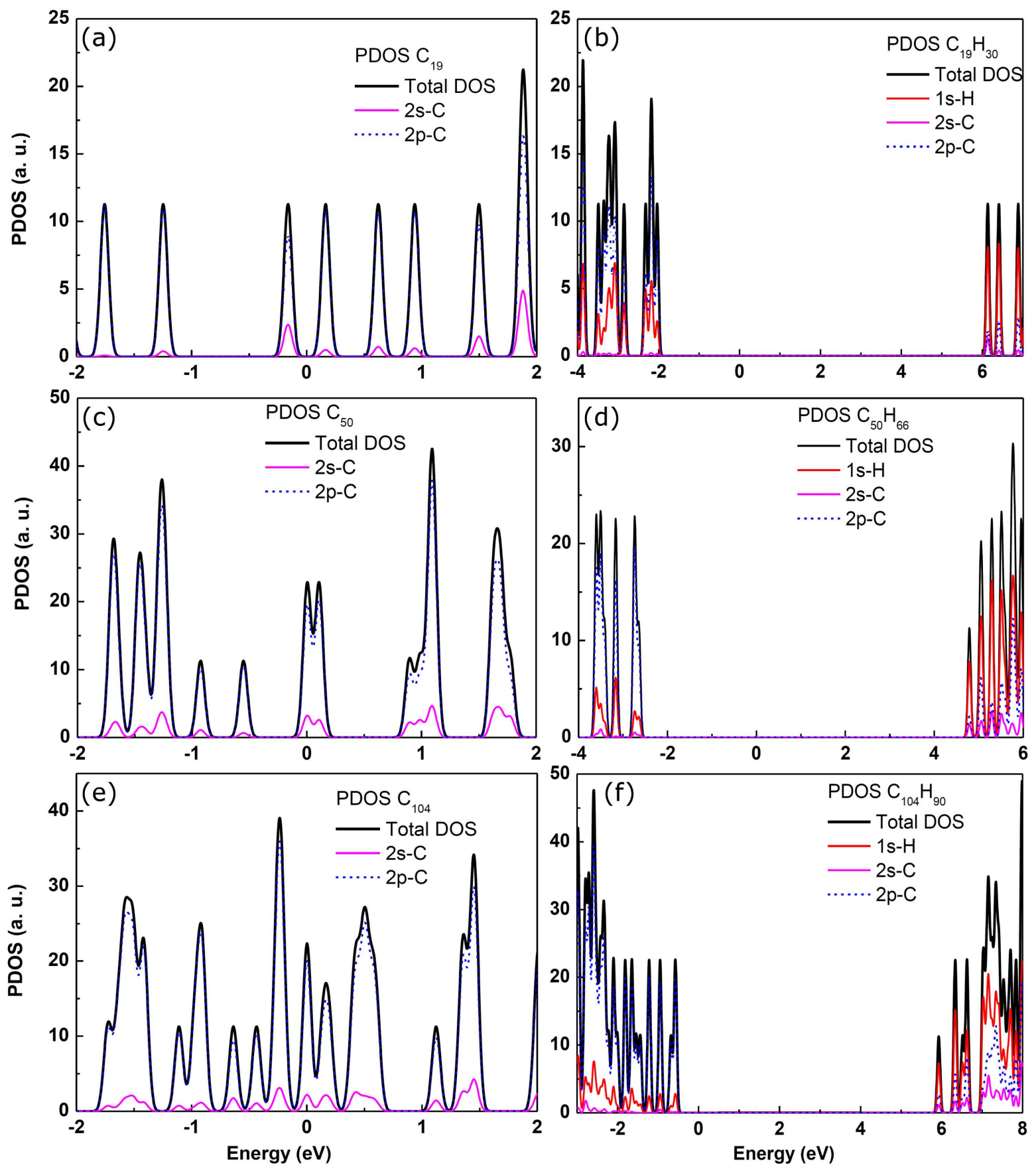

Fig. 4. The total density of states (DOS) of different samples and their corresponding partial density of states (PDOS) analysis. The broadening is $0.05 \mathrm{eV}$.

Diagrams of the density of states (DOS) of all samples are presented in Fig. 4 in which the Fermi level is fixed at the zero point of energy. An extensive differences between DOS of bare and hydrogenated nanodiamonds can be seen (Fig. 4), while almost no considerable gap energy can be spotted in bare nanodiamond samples, the gap energy larger than its corresponding value to the bulk diamond $(5 \mathrm{eV})$ can be observed for the hydrogenated nanodiamonds.
Another size dependence feature which can be figured out considering the DOS of the samples is that, by increasing the number of carbon atoms of samples, and consequently by increasing the size of nanoparticles, the energy states are changed to have more continual and less discrete characteristics. This feature can be elucidated in Fig. 5 which describes the energy levels diagrams. Figure 5 shows that energy levels of nanodiamonds composed of 50 and 104 carbon atoms in both 
bare and hydrogenated samples in which firstly the more discrete characteristics and secondly the increase of the gap energy are exposed as the size of nanoparticles decreases. Although no gap energies were calculated for the bare nanodiamonds of $\mathrm{C}_{50}$ and $\mathrm{C}_{104}$, the sample of $\mathrm{C}_{19}$ revealed gap energy of about $0.33 \mathrm{eV}$. In hydrogenated samples of $\mathrm{C}_{19} \mathrm{H}_{30}, \mathrm{C}_{50} \mathrm{H}_{66}$ and $\mathrm{C}_{104} \mathrm{H}_{90}$, the gap energies are estimated as about $8.2,7.4$, and $6.5 \mathrm{eV}$, respectively which are still higher in comparison to the gap energy of bulk diamond $(5 \mathrm{eV})$. The widening of gap energy is attributed to the quantum confinement effects. Raty et al. [10] indicated that rapid decrease of gap energy by increasing particle size is observed as far as the gap energy coincides with its bulk structure value for particles with diameter of about $1 \mathrm{~nm}$.
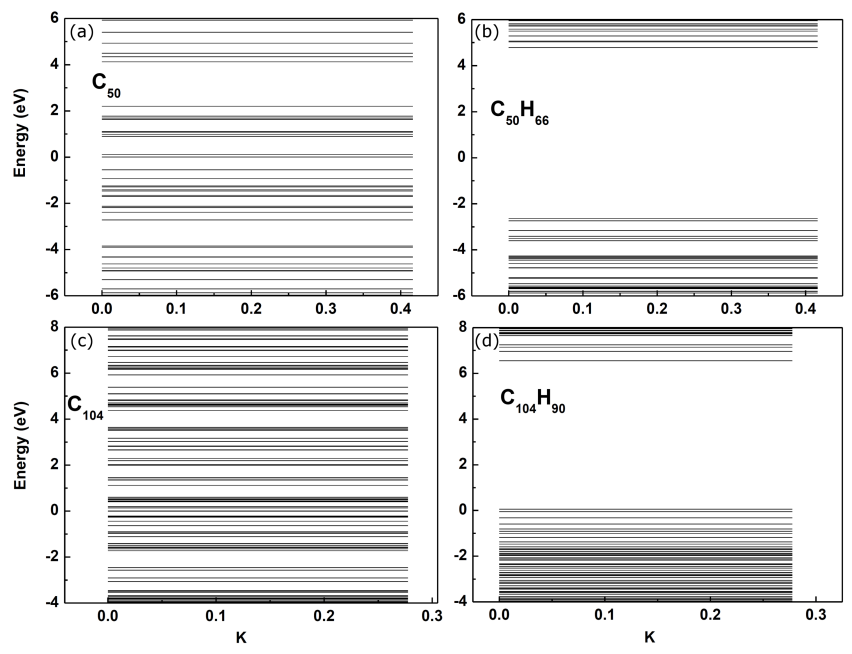

Fig. 5. Energy level diagram of (a) $\mathrm{C}_{50}$, (b) $\mathrm{C}_{50} \mathrm{H}_{66}$, (c) $\mathrm{C}_{104}$ and (d) $\mathrm{C}_{104} \mathrm{H}_{90}$.

In this work, the calculated gap energy for $\mathrm{C}_{104} \mathrm{H}_{90}$ with diameter of about $1.2 \mathrm{~nm}$ was $6.5 \mathrm{eV}$ which is however greater than the gap energy of bulk diamond, denoting that quantum confinement influences the electronic structure of nanodiamond with diameter larger than $1 \mathrm{~nm}$.

In order to investigate the origin of the states around the Fermi level which causes the reduction and also removal of gap energy of hydrogenated and bare nandiamonds, the highest occupied molecular orbital (HOMO) and lowest unoccupied molecular orbital (LUMO) states were calculated and presented in Fig. 6. It can be noticed that HOMO and LUMO states of bare nanodiamonds were located at the surface and interface of the core and the surface of the nanoparticles and contribution of core atoms to these orbitals can be hardly found. In contrast, the HOMO of hydrogenated nanodiamonds was located inside the nanoparticles and LUMO was placed at the surface of hydrogenated ND.

HOMO, LUMO, and gap energies of the samples are summarized in Table I. The size dependent characteristic of HOMO and LUMO of hydrogenated nanodiamonds in
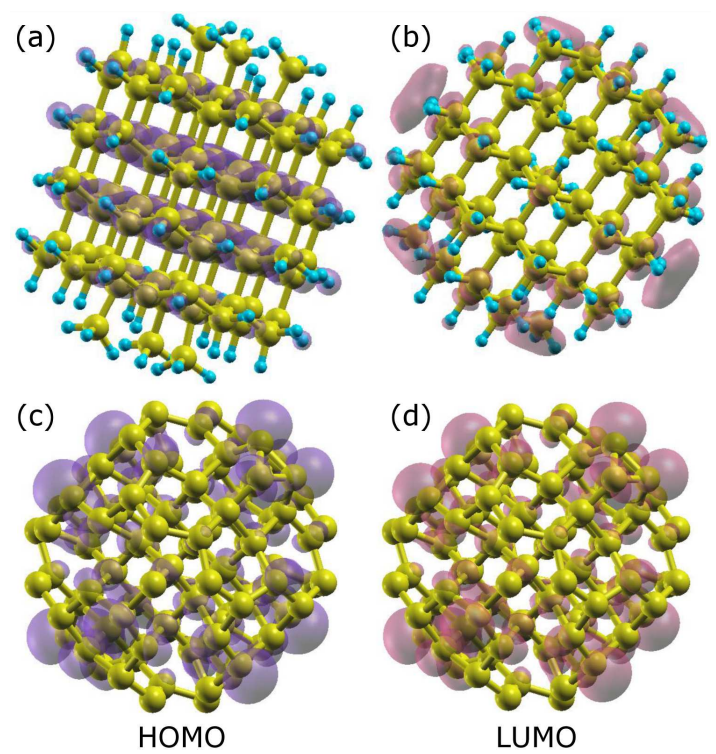

Fig. 6. (a) $\mathrm{HOMO}$ and (b) LUMO of $\mathrm{C}_{104} \mathrm{H}_{90}$ and (c) HOMO and (d) LUMO of $\mathrm{C}_{104}$ when the isovalue is $0.001 \mathrm{e} \AA^{-3}$.

TABLE I

Calculated Fermi, HOMO, LUMO and gap energy of different samples in $\mathrm{eV}$.

\begin{tabular}{l|c|c|c|c|c}
\hline \hline Sample & $E_{\text {Hомо }}$ & $E_{\text {Lumo }}$ & $E_{g}$ & $\begin{array}{c}\text { Electron } \\
\text { affinity }[\mathrm{eV}]\end{array}$ & $\begin{array}{c}\text { Ionization } \\
\text { energy }[\mathrm{eV}]\end{array}$ \\
\hline $\mathrm{C}_{19} \mathrm{H}_{30}$ & -6.04 & 2.13 & 8.19 & -3.82 & 8.02 \\
$\mathrm{C}_{50} \mathrm{H}_{66}$ & -5.30 & 2.13 & 7.43 & -3.53 & 6.87 \\
$\mathrm{C}_{104} \mathrm{H}_{90}$ & -4.61 & 1.89 & 6.50 & -3.05 & 5.96 \\
$\mathrm{C}_{19}$ & -4.47 & -4.14 & 0.33 & &
\end{tabular}

this work, and in agreement to achievements by Wang et al. [12], are entirely different. This difference is due to the origins of these orbitals as stated by Drummond et al. [14]. Since the HOMO of hydrogenated nanodiamonds is located inside the particles, it can contribute into highest valence band upon increasing the particles size to the bulk structure and therefore it can be mentioned that size dependence of HOMO is compatible to the quantum confinement model whereas the LUMO which is located at surface, can be affected by the surface and defect level and can be located under the lowest conduction band of bulk diamond by increasing the particle size [12]. Therefore LUMO does not display quantum confinement [12]. In case of bare diamond both HOMO and LUMO are originated from atoms at the surface or interface of the core and the surface and are less influenced by core atoms.

The partial density of states (PDOS) analysis of the bare nanodiamonds depicted in Fig. 4 reveals that the contribution of $\mathrm{C} 2 p$ is more significant than $\mathrm{C} 2 s$ in HOMO and in LUMO. In hydrogenated samples however the situation is different due to presence of hydrogen. It can be noticed that C $2 p$ has the higher and C $2 s$ 
has lower influence to $\mathrm{HOMO}$ of $\mathrm{C}_{19} \mathrm{H}_{30}, \mathrm{C}_{50} \mathrm{H}_{66}$, and $\mathrm{C}_{104} \mathrm{H}_{90}$, while their LUMO mainly aroused by $\mathrm{H} 1 s$ and C $2 p$ and $\mathrm{C} 2 s$ are in the next significance to the LUMO, respectively.

In order to figure out the presence of quantum confinement effects in sample larger than $1 \mathrm{~nm}$ of the present study, the electron affinity (EA) and ionization potential (IP) of hydrogenated samples are calculated through the following equations:

$$
\begin{aligned}
& \mathrm{EA}=E_{N}-E_{N-1}, \\
& \mathrm{IP}=E_{N}-E_{N+1} .
\end{aligned}
$$

Here $E_{N}$ is defined as the ground state energy of the neutral system including $N$ electrons and $E_{N-1}$ and $E_{N+1}$ are related to the energy of optimized ionic system. The calculated electron affinities and ionization energies are presented in Table I. It can be observed that the hydrogenated nanodiamonds, in agreement to the literature $[14,30]$, show the negative electron affinities (NEA). HOMO and LUMO energies are related to the IP and NEA, respectively. Therefore in accordance to the obtained results, the size dependence of IP $(\approx$ HOMO energy) of the hydrogenated samples can be observed.

Considering Table I, it can be deduced that when the ratio of the number of carbon atoms to the number of hydrogen atoms $(\mathrm{C} / \mathrm{H})$ increases, the NEA of the hydrogenated samples follows the increasing trend. Similar result was reported by Zhang et al. [30].

As it has been stated earlier, the quantum confinement effects on the gap energy of the particles larger than $1 \mathrm{~nm}$ are observed in this work. However, our results are in contrast to the achievements of Drummond et al. [14] and Raty et al. [29], which demonstrated that the quantum confinement effects can be only observed in diamond particles smaller than $1 \mathrm{~nm}$. This controversy can be explained by taking into account of the $\mathrm{C} / \mathrm{H}$ ratio of the samples. Although the $\mathrm{C} / \mathrm{H}$ ratio of the hydrogenated diamond increases as the size of nanoparticles increases, but for a defined number of carbon, the number of hydrogen atoms in our samples are higher compared to the works by Drummond et al. [14] and Raty et al. [29]. The PDOS analysis of the hydrogenated samples (Fig. 4) revealed that the contribution of $\mathrm{H} 1 s$ states to the HOMO are much lower than $\mathrm{C} 2 p$ sates, showing that the $\mathrm{C} 2 p$ states in $\mathrm{C}-\mathrm{H}$ bonds is lower than the $\mathrm{C}-\mathrm{C}$ bond. Therefore, for a sample with specific number of carbon, when the number of hydrogen atoms and consequently number of $\mathrm{C}-\mathrm{H}$ bonds are higher, the HOMO level of the system is lower leading to higher gap energy value [30].

In order to investigate the relative stability of bare and hydrogenated nanodiamond in this work, formation energy for each carbon atoms has been calculated according to the following equation $[11,31]$ :

$$
E_{\text {formation }}=\left[E_{\text {total }}-N_{\mathrm{C}} E_{\mathrm{C}}-\left(N_{\mathrm{H}} / 2\right) E_{\mathrm{H}_{2}}\right] / N_{\mathrm{C}} .
$$

Here $N_{x}$ is the number of carbon or hydrogen atoms, $E_{x}$ is the total energy related to each single carbon atom or hydrogen molecule, and $E_{\text {total }}$ is the total energy of nanodiamond sample.
The calculated formation energies are demonstrated in Table II, it can be found that the hydrogenated nanodiamonds have lower formation energy compared to bare nanodiamonds and consequently have higher relative stability. When the number of carbon atoms of the hydrogenated samples increases, the formation energies are observed to be increasing. According to the results obtained for bare nanodiamonds, although, the formation energy fluctuates when the number of carbon atoms increases, it can be observed that the bare nanodiamond of $\mathrm{C}_{104}$ has lower formation energy compared to $\mathrm{C}_{50}$ and $\mathrm{C}_{19}$, indicating that by increasing the bare nanoparticles diameter, more stability is achieved. These results are in contrast to the size dependent stability of hydrogenated diamond as described in Table II. This achievement is in complementary to what has been estimated by Raty et al. [10]. They predicted the higher stability for the bare nanodiamonds when their diameter was in the range of 2-3 nm. Here in this work, while studying the small nanocluster of diamond ( $>1 \mathrm{~nm}$ ), we found that hydrogenated nanodiamond particles are more stable. This can explain the high colloidal stability and production of individual nanodiamond suspension by hydrogenated nanodiamond which can be further utilized for different applications.

\section{TABLE II}

Nanoparticles diameter, total and formation energies of the studied samples

\begin{tabular}{l|c|c|c}
\hline \hline Sample & $\begin{array}{c}\text { Particle } \\
\text { diameter [nm] }\end{array}$ & $\begin{array}{c}\text { Total } \\
\text { energy [eV] }\end{array}$ & $\begin{array}{c}\text { Formation } \\
\text { energy [eV] }\end{array}$ \\
\hline $\mathrm{C}_{19} \mathrm{H}_{30}$ & 0.79 & -3557.83 & -10.22 \\
$\mathrm{C}_{50} \mathrm{H}_{66}$ & 0.98 & -9153.37 & -10.09 \\
$\mathrm{C}_{104} \mathrm{H}_{90}$ & 1.17 & -18291.24 & -10.01 \\
$\mathrm{C}_{19}$ & 0.42 & -3052.33 & -8.31 \\
$\mathrm{C}_{50}$ & 0.78 & -8024.41 & -8.16 \\
$\mathrm{C}_{104}$ & 0.93 & -16715.89 & -8.40
\end{tabular}

\section{Conclusion}

The results showed that the band gap of hydrogenated nanodiamonds diminishes from 8.2 to $6.5 \mathrm{eV}$ when the size of nanoparticles (number of carbon atoms) is increased indicating that quantum confinement effect is still significant for samples larger than $1 \mathrm{~nm}$. The largest hydrogenated sample in this study with a diameter of about $1.2 \mathrm{~nm}$ has larger energy gap $(6.5 \mathrm{eV})$ compared to the bulk diamond $(5 \mathrm{eV})$. In contrast there was no gap energy for bare nanodiamonds composed by 50 and 104 carbon atoms while the small gap energy of about $0.33 \mathrm{eV}$ was observed for the smallest sample of bare nanodiamond $\left(\mathrm{C}_{19}\right)$. The observed quantum confinement effect of particles larger than $1 \mathrm{~nm}$ was explained considering the electron affinity and the PDOS analysis. Stability study and formation energy calculation revealed that when the size of nanodiamonds is below $1 \mathrm{~nm}$, hydrogenated nanodiamonds are more stable than the bare feature of ND. 


\section{References}

[1] O.A. Shenderova, V.V. Zhirnov, D.W. Brenner, Crit. Rev. Solid State Mater. Sci. 27, 227 (2002).

[2] V.I. Kurmachev, Y.V. Timoshkov, T.I. Orehovkaja, V.Y. Timoshkov, Phys. Solid State 46, 679 (2004).

[3] C. Wang, B. Zheng, W.T. Zheng, Q. Jiang, Diam. Relat. Mater. 17, 204 (2008).

[4] Y.V. Butenko, P.R. Coxon, M. Yeganeh, A.C. Brieva, K. Liddell, V.R. Dhanak, L. Siller, Diam. Relat. Mater. 17, 962 (2008).

[5] A.S. Barnard, S.P. Russo, I.K. Snook, Diam. Relat. Mater. 12, 1867 (2003).

[6] M. Yeganeh, P.R. Coxon, A.C. Brieva, V.R. Dhanak, L. Šiller, Y.V. Butenko, Phys. Rev. B 75, 155404 (2007).

[7] M. Chen, T.P. Straatsma, Z. Fang, D.A. Dixon, J. Phys. Chem. C 120, 20400 (2016).

[8] S. Stehlik, M. Varga, M. Ledinsky, V. Jirasek, A. Artemenko, H. Kozak, L. Ondic, V. Skakalova, G. Argentero, T. Pennycook, J.C. Meyer, A. Fejfar, A. Kromka, B. Rezek, J. Phys. Chem. C 119, 27708 (2015)

[9] Y.K. Chang, H.H. Hsieh, W.F. Pong, M.H. Tsai, F.Z. Chien, P.K. Tseng, L.C. Chen, T.Y. Wang, K.H. Chen, D.M. Bhusari, J.R. Yang, S.T. Lin, Phys. Rev. Lett. 82, 5377 (1999).

[10] J.Y. Raty, G. Galli, Comput. Phys. Commun. 169, $14(2005)$

[11] M.H. Saani, M. Kargarian, A. Ranjbar, Phys. Rev. $B$ 76, 035417 (2007).

[12] C. Wang, B. Zheng, W.T. Zheng, C.Q. Qu, L. Qiao, S.S. Yu, Q. Jiang, Diam. Relat. Mater. 18, 1310 (2009).

[13] J. Jiang, L. Sun, B. Gao, Z. Wu, W. Lu, J. Yang, Y. Luo, J. Appl. Phys. 108, 094303 (2010).

[14] N.D. Drummond, A.J. Williamson, R.J. Needs, G. Galli, Phys. Rev. Lett. 95, 096801 (2005).
[15] V.V. Zhirnov, O.A. Shenderova, D.L. Jaeger, T. Tyler, D.A. Areshkin, D.W. Brenner, J.J. Hren, Phys. Solid State 146, 657 (2004)

[16] D. Tan, S. Zhou, B. Xu, P. Chen, Y. Shimotsuma, K. Miura, J. Qiu, Carbon 62, 374 (2013).

[17] M. Yeganeh, F. Badieian Baghsiyahi, J. Phys. Chem. Solids 124, 235 (2019)

[18] R.P. Fehlhaber, L.A. Bursill, Philos. Mag. B 79, 477 (1999).

[19] P.W. Chen, Y.S. Ding, Q. Chen, F.L. Huang, S.R. Yun, Diam. Relat. Mater. 9, 1722 (2000).

[20] Q. Chen, S. Yun, Mater. Res. Bull. 35, 1915 (2000).

[21] G. Wulff, Z. Krystallogr. Mineral 34, 499 (1901).

[22] A.R. Roosen, R.P. McCormack, W. Craig Carter, Computat. Mater. Sci. 11, 16 (1998).

[23] J.M. Soler, E. Artacho, J.D. Gale, A. Garcia, J. Junquera, P.E.A. Ordejon, J. Phys. Condens. Matter 14, 2745 (2001)

[24] J.P. Perdew, K. Burke, M. Ernzerhof, Phys. Rev. Lett. 77, 3865 (1996).

[25] N. Troullier, J.L. Martins, Phys. Rev. B 43, 1993 (1990).

[26] N. Troullier, J.L. Martins, Solid State Commun. 74 , 613 (1990).

[27] H.J. Monkhorst, J.D. Pack, Phys. Rev. B 13, 5188 (1976).

[28] T.F. Liu, S. Ali, B. Li, D.S. Su, ACS Catal. 7, 3779 (2017).

[29] J.Y. Raty, G. Galli, C. Bostedt, T.V. Buuren, L. Terminello, Phys. Rev. Lett. 90, 037401 (2003).

[30] Z. Zhang, Y. Dai, J. Theor. Computat. Chem. 9, 353 (2010).

[31] G. McIntosh, M. Yoon, S. Berber, D. Tomanek, Phys. Rev. B 70, 045401 (2004) 